PROCEEDINGS OF THE

AMERICAN MATHEMATICAL SOCIETY

Volume 137, Number 10, October 2009, Pages 3235-3246

S 0002-9939(09)09981-X

Article electronically published on June 4, 2009

\title{
VERTEX DECOMPOSABLE GRAPHS AND OBSTRUCTIONS TO SHELLABILITY
}

\author{
RUSS WOODROOFE
}

(Communicated by Jim Haglund)

\begin{abstract}
Inspired by several recent papers on the edge ideal of a graph $G$, we study the equivalent notion of the independence complex of $G$. Using the tool of vertex decomposability from geometric combinatorics, we show that 5chordal graphs with no chordless 4-cycles are shellable and sequentially CohenMacaulay. We use this result to characterize the obstructions to shellability in flag complexes, extending work of Billera, Myers, and Wachs. We also show how vertex decomposability may be used to show that certain graph constructions preserve shellability.
\end{abstract}

\section{INTRODUCTION}

Let $G=(V, E)$ be a graph with vertex set $V=\left\{x_{1}, \ldots, x_{n}\right\}$. The independence complex of $G$, denoted $I(G)$, is the simplicial complex with vertex set $V$ and with faces the independent sets of $G$. When it causes no confusion, we will say that $G$ satisfies some property if its independence complex does. For example, we will say that $G$ is shellable if $I(G)$ is shellable. The independence complex has been previously studied in e.g. 11, 17, 19.

The Stanley-Reisner ring of $I(G)$ is

$$
k\left[x_{1}, \ldots, x_{n}\right] /\left(x_{i} x_{j}: x_{i} x_{j} \in E\right) .
$$

The quotient in the above ring is also called the edge ideal of $G$ and has been an object of study in its own right [26. In particular, a recent series of papers 12, 13, 16, 24 has worked from the edge ideal to show that chordal graphs are sequentially Cohen-Macaulay and shellable and that certain graph constructions preserve shellability and/or being sequentially Cohen-Macaulay.

In this paper, we consider vertex decomposability in graphs. In Section 2, we recall the definition of a vertex decomposable simplicial complex and show what this means for (the independence complexes of) graphs. As an easy consequence we recover the result that chordal graphs are shellable, hence sequentially CohenMacaulay. In Section 3, we give a geometric proof that the only cyclic graphs which are vertex decomposable, shellable and/or sequentially Cohen-Macaulay are $C_{3}$ and $C_{5}$. In Section 4, we prove the main theorem of the paper:

Received by the editors January 8, 2009.

2000 Mathematics Subject Classification. Primary 13F55, 05C38, 05E99.

Key words and phrases. Sequentially Cohen-Macaulay, independence complex, edge ideal, chordal graphs.

(C)2009 American Mathematical Society Reverts to public domain 28 years from publication 
Theorem 1 (Main Theorem). If $G$ is a graph with no chordless cycles of length other than 3 or 5 , then $G$ is vertex decomposable (hence shellable and sequentially Cohen-Macaulay.)

In Section 5, we reinterpret Theorem 1 in terms of obstructions to shellings, answering a question of Wachs. We also give an application to domination numbers, in the style of 19. In Section 6, we examine several graph constructions that preserve vertex decomposability. Finally, in Section 7 we close with some comments on classes of sequentially Cohen-Macaulay graphs.

Note 2. Independence complexes have been studied more extensively in the combinatorics literature as flag complexes [22, Chapter III.4 and references]. Many papers on flag complexes study them by considering the clique complex. We notice that the clique complex of a graph $G$ is the independence complex of the complement graph of $G$.

1.1. Cohen-Macaulay complexes. We review briefly the background definitions from geometric combinatorics and graph theory.

A simplicial complex $\Delta$ is pure if all of its facets (maximal faces) are of the same dimension. A complex $\Delta$ is shellable if its facets fit together nicely. The precise definition will not be important to us, but can be found, with much additional background, in 28, Lecture 3]. The link of a face $F$ in $\Delta$ is

$$
\operatorname{link}_{\Delta} F=\{G: G \cup F \text { is a face in } \Delta, G \cap F=\emptyset\} .
$$

Let $k$ be a field or the ring of integers. A complex $\Delta$ is Cohen-Macaulay over $k$ if $\widetilde{H}_{i}\left(\operatorname{link}_{\Delta} F ; k\right)=0$ for all faces $F$ and $i<\operatorname{dim}\left(\operatorname{link}_{\Delta} F\right)$. More intuitively, a complex is Cohen-Macaulay if it has the homology of a bouquet of top-dimensional spheres and if every link satisfies the same condition. It is a well-known fact that any Cohen-Macaulay complex is pure. Any pure, shellable complex is Cohen-Macaulay over any $k$. Our results will be independent of the choice of $k$, and we henceforth drop it from our notation.

Since simplicial complexes that are not pure are often interesting, we study Stanley's extension [22, Chapter III.2] of the definition of Cohen-Macaulay (and its relationship with shellability) to arbitrary simplicial complexes. The pure $i$-skeleton of $\Delta$ is the complex generated by all the $i$-dimensional faces of $\Delta$. A complex is sequentially Cohen-Macaulay if the pure $i$-skeleton is Cohen-Macaulay for all $i$. Any shellable complex is sequentially Cohen-Macaulay.

$\Delta$ is a Cohen-Macaulay complex if and only if the Stanley-Reisner ring of $\Delta$ is a Cohen-Macaulay ring. There is also a ring-theoretic notion of sequentially CohenMacaulay [22, Definition III.2.9]. For more background, refer to [4] and [22] for the combinatorial point of view or to [8] for a more ring-theoretic approach.

1.2. Chordless paths and cycles. A chordless path of length $n$ in a graph $G$ is a path $v_{1}, v_{2}, \ldots, v_{n}$ in $G$ with no chord, i.e. with no edge $v_{i} v_{j}$ with $j \neq i+1$. Equivalently, the induced graph on $\left\{v_{1}, \ldots, v_{n}\right\}$ is the path on $n$ vertices. In a like manner, a chordless cycle of length $n$ is an induced $n$-cycle.

A graph is $k$-chordal if it has no chordless cycles of length $>k$, and chordal if it is 3 -chordal. 


\section{VERTEX DECOMPOSABILITY AND SHEDDING VERTICES}

A simplicial complex $\Delta$ is recursively defined to be vertex decomposable if it is either a simplex or else has some vertex $v$ so that

(1) both $\Delta \backslash v$ and $\operatorname{link}_{\Delta} v$ are vertex decomposable, and

(2) no face of $\operatorname{link}_{\Delta} v$ is a facet of $\Delta \backslash v$.

A vertex $v$ which satisfies Condition (2) is called a shedding vertex. Vertex decompositions were introduced in the pure case by Provan and Billera 20] and extended to non-pure complexes by Björner and Wachs [5, Section 11].

A vertex decomposable complex is shellable. One proof of this fact is via the following lemma of independent interest:

Lemma 3 (Wachs [27, Lemma 6]). If $\Delta$ is a simplicial complex with shedding vertex $v$ and if both $\Delta \backslash v$ and $\operatorname{link}_{\Delta} v$ are shellable, then $\Delta$ is shellable.

The shelling order in Lemma 3 is that of $\Delta \backslash v$, followed by the facets of $v * \operatorname{link}_{\Delta} v$ in the order of the shelling of link ${ }_{\Delta} v$.

To summarize, we have the chain of implications:

vertex decomposable $\Longrightarrow$ shellable $\Longrightarrow$ sequentially Cohen-Macaulay.

Both implications are known to be strict.

The definition of vertex decomposable (and so of shedding vertex) translates nicely to independence complexes. Let $N(v)$ denote the open neighborhood of $v$, that is, all vertices adjacent to $v$. Let $N[v]$ denote the closed neighborhood of $v$, which is $N(v)$ together with $v$ itself, so that $N[v]=N(v) \cup\{v\}$.

Lemma 4. An independence complex $I(G)$ is vertex decomposable if $G$ is a totally disconnected graph (with no edges) or if

(1) $G \backslash v$ and $G \backslash N[v]$ are both vertex decomposable, and

(2) no independent set in $G \backslash N[v]$ is a maximal independent set in $G \backslash v$.

Proof. Translate the definitions!

A shedding vertex of $G$ is any vertex which satisfies Condition (2) of Lemma 4 , A useful equivalent condition for shedding vertices is:

Condition 5. For every independent set $S$ contained in $G \backslash N[v]$, there is some $x \in N(v)$ so that $S \cup\{x\}$ is independent.

We make a first observation:

Lemma 6. If $N[v] \subseteq N[w]$ (so that in particular $v$ and $w$ are adjacent), then $w$ is a shedding vertex for $G$.

Proof. Since every neighbor of $v$ is also a neighbor of $w$, there are no edges from $v$ to any vertex of $G \backslash N[w]$. Thus, any $v$ can be added to any independent set in $G \backslash N[w]$ while preserving independence.

Recall that a simplicial vertex is a vertex $v$ such that $N[v]$ is a clique. A wellknown theorem of Dirac [21, Theorem 6.3] says that every chordal graph has a simplicial vertex. Thus, we have:

\section{Corollary 7.}

(1) Any neighbor of a simplicial vertex is a shedding vertex for $G$. 
(2) A chordal graph is vertex decomposable (hence shellable [24, Theorem 1.2] and sequentially Cohen-Macaulay [13, Theorem 1.2]; also closely related is [19, Theorem 1.4]).

Proof. If $v$ is a simplicial vertex and $w$ is a neighbor of $v$, then $N[v] \subseteq N[w]$, and Lemma 6 gives (1). For (2), the theorem of Dirac thus says that a chordal graph has a shedding vertex $w$ if it is not totally disconnected. Since every induced subgraph of a chordal graph is chordal, both $G \backslash w$ and $G \backslash N[w]$ are inductively vertex decomposable.

Remark 8. Vertices satisfying the condition of Lemma 6 have been studied before under the name dominant vertices, in the context of so-called dismantlable graphs 15. 6. However, dismantlability is a tool for understanding the homotopy type of the clique complex of $G$, i.e., for understanding the independence complex of the complement of $G$. Since $v$ and $w$ will not be adjacent in the complement, there does not seem to be any direct interpretation of dismantlability in terms of vertex decomposability.

Remark 9. Anton Dochtermann and Alexander Engström also examined vertex decomposability in graphs, independently and at about the same time [11, Section 4] as the author did. In particular, they prove Corollary 7 , and a special case of Proposition 22, they also notice that the result of Billera and Myers discussed in Section 5.1 is a special case of Corollary 7

\section{CYCLIC GRAPHS}

Corollary 7 (2) states that if $G$ has no chordless cycles of length greater than 3 , then it is vertex decomposable. Let $C_{n}$ be the cyclic graph on $n$ vertices. We discuss a partial converse:

Theorem 10 (Francisco/Van Tuyl [13, Proposition 4.1]). $C_{n}$ is vertex decomposable/shellable/sequentially Cohen-Macaulay if and only if $n=3$ or 5 .

Theorem 10 was proved with algebraic techniques in [13, Proposition 4.1]. We give a geometric proof here.

We start with a technical lemma:

Lemma 11. Let $n=2 r+1,0<d<r$. Let the $d$-dimensional complex $\Delta_{n}^{d}$ be the complex with vertex set $\mathbb{Z} / n \mathbb{Z}$ and with facets $F_{i}=\{i, i+2, \ldots, i+2 d\}$ for $i=1, \ldots, n$. Then $\Delta_{n}^{d} \cong S^{1}$.

Proof. Consider $\Delta_{n}^{d}$ for $d>1$. A facet $F_{i}$ has codimension 1 intersection with two other facets: $F_{i-2}$ and $F_{i+2}$. Since $d>1$, and since all codimension 1 faces of $F_{i}$ other than $F_{i} \cap F_{i-2}$ and $F_{i} \cap F_{i+2}$ are "free" (contained in a unique facet), we can collapse $F_{i}$ onto $F_{i} \cap F_{i-2}$ and $F_{i} \cap F_{i+2}$. More formally, the face $\{i, i+2 d\} \subset F_{i}$ is free, so we can remove all faces containing $\{i, i+2 d\}$ via an elementary collapse [3. Section 11.1], which preserves homotopy type. Every face $F$ not containing $\{i, i+2 d\}$ is in either $F_{i} \cap F_{i+2}$ (if $\left.i \notin F\right)$ or $F_{i} \cap F_{i-2}$ (if $\left.i+2 d \notin F\right)$.

Performing a similar collapse at each $F_{i}$ leaves us a simplicial complex with facets $F_{i} \cap F_{i-2}$ for $i=1, \ldots, n$. But $F_{i} \cap F_{i-2}=\{i, \ldots, i+2 d-2\}$, and we see that we have collapsed $\Delta_{n}^{d}$ to $\Delta_{n}^{d-1}$. Thus, $\Delta_{n}^{d} \cong \Delta_{n}^{d-1}$ when $d>1$.

Since $n$ is odd, repeatedly adding 2 to some $i \in \mathbb{Z} / n \mathbb{Z}$ will cover all vertices; hence $\Delta_{n}^{1}$ is the 1 -complex $C_{n} \cong S^{1}$. 


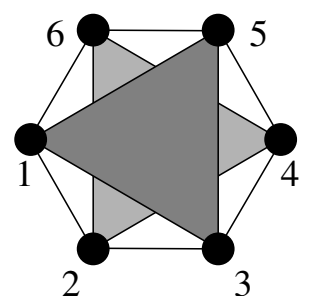

Figure 3.1. The pure 2-skeleton of $I\left(C_{6}\right)$ has two disconnected faces.

Proof of Theorem 10. The if direction is easy: the independence complex of $C_{3}$ is three disconnected vertices, while that of $C_{5}$ is $C_{5}$ as a 1-complex. Both are clearly vertex decomposable.

In the other direction, we show that the pure $d$-skeleton is not Cohen-Macaulay, where $d$ is the top dimension of the complex (i.e., $d=\operatorname{dim} I\left(C_{n}\right)$ ). There are two cases, based on whether $n$ is even or odd. (It may be helpful to look at Figures 3.1 and 3.2 while reading the following.)

Case 1. $n=2 r$. Then the top-dimensional facets have dimension $r-1$, and there are two of them: one with all even vertices, the other with all odd vertices. As the pure $r-1$ skeleton is not even connected, it is certainly not Cohen-Macaulay.
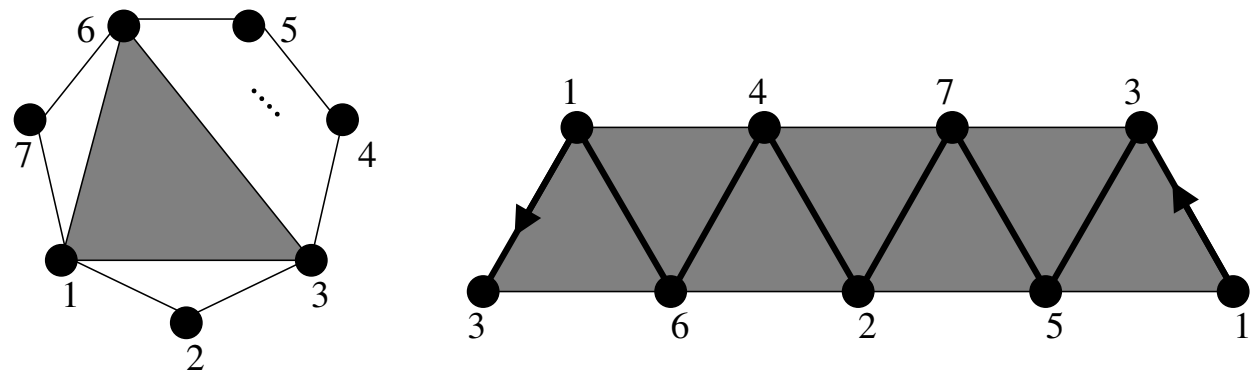

Figure 3.2. $I\left(C_{7}\right)$ is the Möbius strip. The dark line shows the subcomplex $\Delta_{7}^{1}$.

Case 2. $n=2 r+1$, where $r \geq 2$. Then the top-dimensional facets once more have dimension $r-1$. All such facets are obtained by taking a sequence of $r$ alternating vertices in $C_{n}$, with one skip of 2 vertices. We see that the top-dimensional skeleton of $I\left(C_{n}\right)$ is the complex $\Delta_{n}^{r-1}$ discussed in Lemma 11, and so homotopic to $S^{1}$. Thus, the pure $r-1$ skeleton is Cohen-Macaulay only when $r-1=1$, i.e., when $n=5$.

Since every pure skeleton of a sequentially Cohen-Macaulay complex is CohenMacaulay, we have shown that $C_{n}$ is not sequentially Cohen-Macaulay (hence not shellable or vertex decomposable) for $n \neq 3,5$.

Example 12. The pure 2-skeleton of $I\left(C_{6}\right)$ consists of two disconnected triangles, as shown in Figure 3.1, while $I\left(C_{7}\right)$ is a (pure) triangulation of the Möbius strip, as seen in Figure 3.2. Lemma 11 collapses $I\left(C_{7}\right)$ to the cycle $1,3,5,7,2,4,6$. 
Remark 13. We computed the homotopy type of the top-dimensional skeleton of $I\left(C_{n}\right)$. The homotopy type of the entire complex $I\left(C_{n}\right)$ was calculated by Kozlov [18, Proposition 5.2].

\section{Proof of MAIN THEOREM}

The previous two sections motivate the result of Theorem 1 In this section, we will give a proof.

A simplicial $k$-path in $G$ is a chordless path $v_{1}, v_{2}, \ldots, v_{k}$ which cannot be extended on both ends to a chordless path $v_{0}, v_{1}, \ldots, v_{k}, v_{k+1}$ in $G$. Thus, a simplicial vertex is a simplicial 1-path.

Chvátal, Rusu, and Sritharan [9] proved a nice generalization of Dirac's Theorem to $(k+2)$-chordal graphs using simplicial $k$-paths. The following lemma of theirs will allow us to use the 5-chordal structure of $G$.

Lemma 14 (Chvátal, Rusu, and Sritharan [9, Lemma 3]). If $G$ is a 5-chordal graph and $G$ contains a chordless 3-path $P_{3}$, then $G$ contains a simplicial 3-path.

Note 15. From a geometric combinatorics point of view, it might make more sense to count edge length and have the above definition be a simplicial $(k-1)$-path, so that a simplicial vertex would be a simplicial 0-path. However, to avoid confusion, I have kept the original, more graph-theoretic definition.

We also need to use the lack of chordless 4-cycles:

Lemma 16. Let $w_{1}, v, w_{2}$ be a simplicial 3-path which is not a subgraph of any chordless $C_{4}$ in $G$. Then $v$ is a shedding vertex.

Proof. We first notice that, since there is no edge $w_{1} w_{2}$, that any $z$ adjacent to both $w_{1}$ and $w_{2}$ must also be adjacent to $v$. Otherwise, $w_{1}, v, w_{2}, z$ would be a chordless 4-cycle.

Suppose by contradiction that $v$ is not a shedding vertex. Then by Lemma 4 and Condition 5 , there is an independent set in $G \backslash N[v]$ which contains a vertex $z_{1}$ adjacent to $w_{1}$, and a vertex $z_{2}$ adjacent to $w_{2}$. Since $z_{1}, z_{2} \in G \backslash N[v]$, neither is adjacent to $v$. No $z$ in $G \backslash N[v]$ is adjacent to both $w_{1}$ and $w_{2}$, so $z_{1}$ is not adjacent to $w_{2}$, and $z_{2}$ is not adjacent to $w_{1}$. Since $z_{1}$ and $z_{2}$ are in an independent set, $z_{1}$ is not adjacent to $z_{2}$.

Counting non-adjacent pairs of vertices, we have just shown that $z_{1}, w_{1}, v, w_{2}, z_{2}$ is a chordless path, which contradicts the definition of simplicial 3-path. Thus $v$ is a shedding vertex, as desired.

Proof of Theorem 1. If $G$ is chordal, then $G$ is vertex decomposable, as shown in Corollary 7 Otherwise, $G$ has some chordless 5 -cycle, hence a chordless 3-path, and by Lemma 14 a simplicial 3-path. Lemma 16 shows that the middle vertex of any simplicial 3-path in $G$ is a shedding vertex, and so by induction $G$ is vertex decomposable.

\section{Applications}

5.1. Obstructions. An obstruction to shellability is a non-shellable complex, all of whose proper subcomplexes are shellable. Thus, any non-shellable complex must contain at least one obstruction to shellability, while a shellable complex may or may not contain some obstructions to shellability as proper subcomplexes. 
The order complex of a poset is the simplicial complex with vertex set the elements of the poset and with face set the chains of comparable elements. Thus, the order complex of $P$ is the independence complex of the incomparability graph on $P$, which puts an edge between two elements if they are incomparable.

The study of obstructions to shellability was initiated by Billera and Myers, with the following theorem:

Theorem 17 (Billera and Myers [2, Cor. 1]). If $P$ is a non-shellable poset, then $P$ contains an induced subposet isomorphic to the poset $D=\{$ two disjoint edges $\}$.

Equivalently, the unique obstruction to shellability in a poset is $D$. We note that the incomparability graph of $D$ is $C_{4}$. Gallai gave a forbidden subgraph characterization of incomparability graphs of posets in [14] (translated to English in 21, Chapter 3]; a more accessible version of the list is in [23, Chapter 3.2]). The forbidden subgraphs include $C_{n}$ for $n \geq 5$. Thus, Theorem 17 follows from Corollary 7 (2).

Wachs studied obstructions to shellability further in [27, where she asked about the obstructions to shellability in a flag complex. Theorem 1 gives a classification, which we summarize in the following theorem:

Theorem 18. The obstructions to shellability in flag complexes are exactly the independence complexes of $C_{n}$, where $n=4$ or $n \geq 6$.

Proof. By Theorem 1, any non-shellable graph $G$ has an induced subgraph (hence subcomplex) isomorphic to such a $C_{n}$. In Theorem 10 we showed that such $C_{n}$ are not shellable, but any proper induced subgraph of $C_{n}$ is chordal, hence shellable.

A natural question suggested by Theorem 18 is whether there is some similar characterization of obstructions to shellability in non-flag complexes, where the minimal non-faces form a hypergraph. One might be led to ask whether the hypergraph of minimal non-faces is always cyclic in an obstruction to shellability. However, examples studied by Wachs 27. show this is not so, as follows.

Let $M_{n}$ be the simplicial complex with faces $\{1,2,3\},\{2,3,4\}, \ldots,\{n-1, n, 1\}$, $\{n, 1,2\}$. In [27, Lemma 5], Wachs shows that $M_{5}, M_{6}$, and $M_{7}$ are obstructions to shellability.

Inspection verifies that $M_{7}$ is a flag complex, in fact that $M_{7}=I\left(C_{7}\right)$. The complexes $M_{5}$ and $M_{6}$ are not flag. The minimal non-faces of $M_{5}$ are $\{1,2,4\},\{2,3,5\}$, $\{3,4,1\},\{4,5,2\},\{5,1,3\}$, which is a cyclic hypergraph, insofar as there is an alternating sequence of edges $e$ and vertices $v \in e$ which visits each edge and vertex exactly once. However, the minimal non-faces of $M_{6}$ are $\{1,4\},\{2,5\},\{3,6\}$ and $\{1,3,5\},\{2,4,6\}$, as pictured in Figure 5.1. I can see no natural generalization of cyclic graph which applies directly to this hypergraph. Interestingly, however, there is an indirect relationship: the edges are the facets of $I\left(C_{6}\right)$.

5.2. Domination numbers. A set $S \subseteq V$ is a dominating set if $\bigcup_{s \in S} N[s]=$ $V$. The dominating number of $G$, denoted $\gamma(G)$, is the minimum cardinality of a dominating set. Meshulam showed [19, Theorem 1.2 (iii)] that the homology of $I(G)$ vanishes below dimension $\gamma(G)-1$ when $G$ is a chordal graph.

We generalize this result in two respects. Let $i(G)$ be the independent domination number, that is, the minimum cardinality of a maximal independent set. Any maximal independent set is a minimal dominating set, so $\gamma(G) \leq i(G)$. 


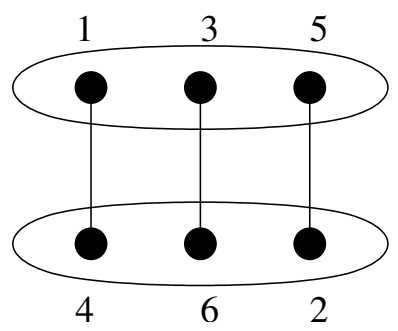

Figure 5.1. The minimal non-face hypergraph of $M_{6}$.

Since a sequentially Cohen-Macaulay complex has homology vanishing below the dimension of the smallest facet, and since the smallest facet of $I(G)$ has cardinality $i(G)$, an immediate consequence is the following:

Corollary 19. If $G$ is any sequentially Cohen-Macaulay graph (over $k$ ), then $\tilde{H}_{j}(I(G) ; k)=0$ for any $j<i(G)-1$.

In particular, we recover the result [19, Theorem 1.2 (iii)] for chordal graphs and $\gamma(G)$ and extend it to a larger class of graphs and a larger graph invariant.

\section{Graph constructions}

We now give examples of how shedding vertices can be used to show that certain graph constructions respect shellability.

Lemma 20. If $G=G_{1} \dot{\cup} G_{2}$, then $I(G)=I\left(G_{1}\right) * I\left(G_{2}\right)$, the join of simplicial complexes. Hence $G$ is vertex decomposable, shellable and/or sequentially CohenMacaulay if and only if $G_{1}$ and $G_{2}$ are.

Proof. It is obvious from the definition that $I(G)$ is the given join and that the join is vertex decomposable if and only if both $I\left(G_{1}\right)$ and $I\left(G_{2}\right)$ are. That the join of two complexes is shellable or sequentially Cohen-Macaulay if and only if both complexes are is well known and can be found for example in 28. (Part of Lemma 20 can be found as [24, Lemma 2.4].)

Example 21. Adding a single vertex to $G$ via a disjoint union forms a cone over $I(G)$. Adding on the graph consisting of two vertices connected by an edge via disjoint union corresponds to taking the suspension of $I(G)$. Thus, for example, the union of $n$ disjoint edges is homotopic to $S^{n-1}$.

Francisco and Hà [12, following Villarreal [25, Theorem 2.2], define a whisker in a graph as a vertex of degree 1. A similar idea seems to be studied in the wider graph theory literature under the name of pendant. We will prefer the latter term here. In [12] and 24], it is shown that, speaking broadly, adding pendants to graphs has good properties for maintaining shellability and the sequentially Cohen-Macaulay property. Their construction essentially works because adding a pendant adds a simplicial vertex. We give an obvious generalization: 
Proposition 22. Let $G_{0}$ be a graph with a complete subgraph $K$, and let $G$ be obtained from $G_{0}$ by adding a new vertex $v$ with edges to all vertices of $K$. (That is, let $G$ be obtained from $G_{0}$ by "starring $K$ ".) Then any element of $K$ is a shedding vertex in $G$; conversely, $G$ is shellable (sequentially Cohen-Macaulay) only if $G_{0} \backslash K$ is.

Proof. Since $N[v]=K \cup\{v\}$, we have that $v$ is a simplicial vertex; hence any neighbor is a shedding vertex (Corollary 7). For the converse statement, we recall that links in a shellable/sequentially Cohen-Macaulay complex have the same property and notice that $\operatorname{link}_{I(G)} v=G \backslash N[v]=G_{0} \backslash K$.

The "clique-starring" construction described in Proposition 22 adds a pendant when $|K|=1$. Whatever the size of $K$, the construction adds a simplicial vertex to $G$. We now consider a construction analogous to a pendant which adds a 3simplicial path.

Proposition 23. Let $G_{0}$ be a graph with a complete subgraph $K$, and let $K_{1}, K_{2}$ be disjoint subgraphs of $K$. Let $G$ be obtained from $G_{0}$ by adding new vertices $w_{1}$, $w_{2}$, and $v$, with $w_{1}$ adjacent to all vertices of $K_{1}, w_{2}$ adjacent to all vertices of $K_{2}$, and $v$ adjacent to $w_{1}$ and $w_{2}$. Then $v$ is a shedding vertex of $G$. Conversely, $G$ is shellable (sequentially Cohen-Macaulay) only if $G_{0}$ is.

Proof. By definition, the path $w_{1}, v, w_{2}$ is 3 -simplicial, while $v$ is in no chordless 4-cycles because $K_{1}$ and $K_{2}$ are disjoint. Lemma 16 shows that $v$ is a shedding vertex. Conversely, $\operatorname{link}_{I(G)} v=G_{0}$, and any link in a shellable/sequentially CohenMacaulay complex has the same property.

We notice in passing that $G \backslash v=G_{0} \cup\left\{w_{1}, w_{2}\right\}$ is formed from $G_{0}$ by performing the construction of Proposition 22 on $K_{1}$ and $K_{2}$.

Another graph construction is that of twinning. If two vertices $v$ and $w$ have the same neighbors, i.e., if $N(w)=N(v)$, then we say $v$ and $w$ are true twins if there is an edge $v w$, and false twins otherwise. There are corresponding graph constructions: add a new vertex $w$ to $G$, together with edges to all neighbors of some $v$. The family of distance hereditary graphs can be defined as the graphs that can be built from a single vertex by adding pendants, true twins, and false twins [7. Chapter 11.6]; twins are also useful in proofs of the Perfect Graph Theorem [10, Chapter 5.5].

Proposition 24. If $v$ and $w$ are true twins, then $v$ and $w$ are shedding vertices.

Proof. We note that $N[v]=N[w]$, and thus by Lemma 6 they are both shedding vertices.

False twins are never shedding vertices, since any maximal independent set including $w$ in $G \backslash N[v]$ is also maximal in $G \backslash v$.

Example 25. The 4-cycle is obtained by adding a false twin of the middle vertex in a 3-path. Thus, adding a false twin to a sequentially Cohen-Macaulay graph can result in a non-sequentially Cohen-Macaulay graph.

One more family of graph operations which has frequently been studied is that of graph products. There are a large number of such operations, based on different rules for putting edges on the Cartesian product of the vertex set. We cannot 
examine all of them, but note that the commonly considered operations of direct product and Cartesian product of graphs do not respect shellability or the sequential Cohen-Macaulay property, for the Cartesian product of two edges (shellable) is a 4-cycle (not sequentially Cohen-Macaulay), while the direct product of an edge with a 3-cycle (both shellable) is the complete bipartite graph $K_{3,3}$, which 24 , Corollary 3.11] shows is not sequentially Cohen-Macaulay.

\section{A COMMENT ON PERFECT GRAPHS}

Herzog, Hibi, and Zheng [16] point out that classifying sequentially CohenMacaulay graphs is likely an intractable problem. We recall their argument. If $\Delta$ is a simplicial complex, then the order complex of the face lattice of $\Delta$ is a flag complex, and it is sequentially Cohen-Macaulay if and only if $\Delta$ is. (The order complex of the face lattice is the barycentric subdivision of $\Delta$.) Herzog, Hibi and Zheng conclude that characterizing sequentially Cohen-Macaulay graphs is as difficult as characterizing all sequentially Cohen-Macaulay complexes. The closely related property of shellability is likely of a similar difficulty.

As we have seen, however, there are families of graphs in which classifying the sequentially Cohen-Macaulay members of the family is possible. That all chordal graphs are sequentially Cohen-Macaulay (Corollary 7) is an example of this type of classification, as is the recursive characterization of sequentially Cohen-Macaulay bipartite graphs in [24, Corollary 3.11]. Other families of graphs may also have interesting answers.

We notice that the argument of Herzog, Hibi, and Zheng can help indicate the families of graphs in which we can hope for such a classification. For example, a perfect graph is one where every induced subgraph has chromatic number equal to the size of its largest clique. The Strong Perfect Graph Theorem says that a graph $G$ is perfect if and only if there are no chordless odd cycles of length $\geq 5$ in either $G$ or its complement. Another fundamental result is that the complement of a perfect graph is also perfect. See 21] for more information and references about perfect graphs. Both chordal graphs and bipartite graphs are perfect, and characterizing the shellability and/or sequential Cohen-Macaulay connectivity of their common super-family would seem like a reasonable aim.

Unfortunately for this aim, poset (in)comparability graphs are perfect, as can be proved either by the direct argument of coloring elements by their rank, or else from Gallai's previously mentioned characterization of poset comparability graphs, which lack odd cycles of length $\geq 5$ [14. Moreover, a complex is sequentially Cohen-Macaulay if and only if its face poset is sequentially Cohen-Macaulay. Thus, characterizing the sequentially Cohen-Macaulay perfect graphs is at least as hard as characterizing which complexes in general are sequentially Cohen-Macaulay.

Considering the intersection of a graph family $\mathcal{F}$ with the family of poset incomparability graphs is a recommended exercise before looking for shellings of graphs in $\mathcal{F}$.

\section{ACKNOWLEDGEMENTS}

I would like to thank Chris Francisco and John Shareshian for helpful references and stimulating discussions. 


\section{REFERENCES}

[1] R. Aharoni, E. Berger, and R. Meshulam, Eigenvalues and homology of flag complexes and vector representations of graphs, Geom. Funct. Anal. 15 (2005), no. 3, 555-566. MR2221142 (2007b:05148)

[2] Louis J. Billera and Amy N. Myers, Shellability of interval orders, Order 15 (1998/99), no. 2, 113-117. MR 1695087 (2000b:06010)

[3] Anders Björner, Topological methods, Handbook of combinatorics, Vols. 1, 2, Elsevier, Amsterdam, 1995, pp. 1819-1872. MR1373690 (96m:52012)

[4] Anders Björner, Michelle Wachs, and Volkmar Welker, On sequentially Cohen-Macaulay complexes and posets, Israel J. Math. 169 (2009), 295-316. MR2460907

[5] Anders Björner and Michelle L. Wachs, Shellable nonpure complexes and posets. II, Trans. Amer. Math. Soc. 349 (1997), no. 10, 3945-3975. MR.1401765 (98b:06008)

[6] Romain Boulet, Etienne Fieux, and Bertrand Jouve, Simplicial simple-homotopy of flag complexes in terms of graphs, arXiv:0809.1751. To appear in European Journal of Combinatorics.

[7] Andreas Brandstädt, Van Bang Le, and Jeremy P. Spinrad, Graph classes: A survey, SIAM Monographs on Discrete Mathematics and Applications, Society for Industrial and Applied Mathematics (SIAM), Philadelphia, PA, 1999. MR.1686154(2001h:05001)

[8] Winfried Bruns and Jürgen Herzog, Cohen-Macaulay rings, Cambridge Studies in Advanced Mathematics, vol. 39, Cambridge University Press, Cambridge, 1993. MR.1251956 (95h:13020)

[9] Vašek Chvátal, Irena Rusu, and R. Sritharan, Dirac-type characterizations of graphs without long chordless cycles, Discrete Math. 256 (2002), no. 1-2, 445-448. MR1927566|(2003g:05106)

[10] Reinhard Diestel, Graph theory, third ed., Graduate Texts in Mathematics, vol. 173, SpringerVerlag, Berlin, 2005. MR2159259 (2006e:05001)

[11] Anton Dochtermann and Alexander Engström, Algebraic properties of edge ideals via combinatorial topology, Electron. J. Combin. 16 (2009), no. 2, Research Paper 2, approx. 24 pp. (electronic).

[12] Christopher A. Francisco and Huy Tài Hà, Whiskers and sequentially Cohen-Macaulay graphs, J. Combin. Theory Ser. A 115 (2008), no. 2, 304-316. MR2382518 (2008j:13050)

[13] Christopher A. Francisco and Adam Van Tuyl, Sequentially Cohen-Macaulay edge ideals, Proc. Amer. Math. Soc. 135 (2007), no. 8, 2327-2337 (electronic). MR2302553 (2008a:13030)

[14] T. Gallai, Transitiv orientierbare Graphen, Acta Math. Acad. Sci. Hungar. 18 (1967), 25-66. MR0221974 (36:5026)

[15] John Ginsburg, Dismantlability revisited for ordered sets and graphs and the fixed-clique property, Canad. Math. Bull. 37 (1994), no. 4, 473-481. MR.1303674(95k:05151)

[16] Jürgen Herzog, Takayuki Hibi, and Xinxian Zheng, Cohen-Macaulay chordal graphs, J. Combin. Theory Ser. A 113 (2006), no. 5, 911-916. MR2231097(2007b:13042)

[17] Caroline J. Klivans, Threshold graphs, shifted complexes, and graphical complexes, Discrete Math. 307 (2007), no. 21, 2591-2597. MR2359603 (2008j:05373)

[18] Dmitry N. Kozlov, Complexes of directed trees, J. Combin. Theory Ser. A 88 (1999), no. 1, 112-122. MR.1713484 (2000j:05036)

[19] Roy Meshulam, Domination numbers and homology, J. Combin. Theory Ser. A 102 (2003), no. 2, 321-330. MR1979537 (2004c:05144)

[20] J. Scott Provan and Louis J. Billera, Decompositions of simplicial complexes related to diameters of convex polyhedra, Math. Oper. Res. 5 (1980), no. 4, 576-594. MR593648 (82c:52010)

[21] Jorge L. Ramírez Alfonsín and Bruce A. Reed (eds.), Perfect graphs, Wiley-Interscience Series in Discrete Mathematics and Optimization, John Wiley \& Sons Ltd., Chichester, 2001. MR1858793 (2002d:05001)

[22] Richard P. Stanley, Combinatorics and commutative algebra, second ed., Progress in Mathematics, vol. 41, Birkhäuser Boston Inc., Boston, MA, 1996. MR1453579 (98h:05001)

[23] William T. Trotter, Combinatorics and partially ordered sets, Dimension theory, Johns Hopkins Series in the Mathematical Sciences, Johns Hopkins University Press, Baltimore, MD, 1992. MR1169299 (94a:06001)

[24] Adam Van Tuyl and Rafael H. Villarreal, Shellable graphs and sequentially Cohen-Macaulay bipartite graphs, J. Combin. Theory Ser. A 115 (2008), no. 5, 799-814. MR2417022 (2009b:13056) 
[25] Rafael H. Villarreal, Cohen-Macaulay graphs, Manuscripta Math. 66 (1990), no. 3, 277-293. MR:1031197 (91b:13031)

[26] Monomial algebras, Monographs and Textbooks in Pure and Applied Mathematics, vol. 238, Marcel Dekker Inc., New York, 2001. MR.1800904 (2002c:13001)

[27] M. L. Wachs, Obstructions to shellability, Discrete Comput. Geom. 22 (1999), no. 1, 95-103. MR:1692690 (2000k:52011)

[28] Michelle L. Wachs, Poset topology: Tools and applications, Geometric combinatorics, IAS/Park City Math. Ser., vol. 13, Amer. Math. Soc., Providence, RI, 2007, pp. 497-615. MR2383132

Department of Mathematics, Washington University in St. Louis, St. Louis, Missouri 63130

E-mail address: russw@math.wustl.edu 